Case Report

\title{
TAKE THE RISK OR LOSE THE CHANCE! CASE REPORT
}

\author{
G. Tsvetkova*, Y.Zdravkov, A. Oscar \\ Department of Ophthalmology, Medical University - Sofia, Bulgaria \\ Clinic of Ophthalmology, Aleksandrovska University Hospital - Sofia, Bulgaria
}

\begin{abstract}
The hallmark of keratoconus is central or paracentral stromal thinning, accompanied by apical protrusion and irregular astigmatism. Patients typically experience variable reductions in visual acuity, image distortion, and increased sensitivity to glare and light. The latter becomes of major interest when patients with keratoconus present with a cataract which contributes to a further visual decline.

PURPOSE: To present a case report of a patient who underwent cataract surgery, with implantation of an intraocular implant called pinhole and correction of an irregular corneal astigmatism as a result of keratoconus.

METHODS: A report of a rare case, the first implantation of XtraFocus implant in Bulgaria for the correction of keratoconus combined with cataract.

RESULTS: All necessary measurements were performed using an iTrace aberrometer and IOL Master 700. The cataract removal was performed by standard phacoemulsification followed by implantation of a pinhole implant in the capsular bag. No complications related to the position of the implant were observed in the postoperative period. Optimal correction of the distance vision and near vision was achieved, and the results were monitored within six months after surgery.

CONCLUSION: The pinhole implant was securely positioned in the capsular bag. Well tolerated by the patient, the implant played a key role in effectively overcoming the irregular corneal astigmatism. There is a significant improvement in visual acuity with results satisfying the patient's high expectations.
\end{abstract}

Key words: stenopeic principle, pinhole, XtraFocus, irregular astigmatism, cataract, iTrace

\section{INTRODUCTION}

Ophthalmology, as a constantly evolving branch of medicine, has greatly benefitted from the technological advances over the recent years. There has been an increasing interest in the correction of irregular corneal astigmatism, leading to the invention and penetration of small hole implants. It is well known that the pinhole effect is based on the stenopeic principle. It is also believed that

Correspondence to: Galateya Toshkova

Tsvetkova, 1 Sv. Georgi Sofiyski Blvd., 1431 Sofia, Bulgaria, +359883770073,

dr.gtsvetkova@gmail.com
Mozi (Mo-Ti), a Chinese philosopher from the $\mathrm{V}$ century $\mathrm{BC}$, had described it for the first time, and Leonardo da Vinci took advantage of it for developing his innovative inventions.

The pinhole is a small circular opening (1), which reduces the beam of light entering through it, eliminates the peripheral aberrations (2), increases the length of the focus and improves the uncorrected visual acuity for distance vision and near vision. This way the image is clarified, but the possible side effects should not be neglected, such as induction of the diffraction phenomenon, reduction of the contrast sensitivity, limitation of the visual field, etc. 
According to a study published in 1977 whose aim to monitor the effect of pinhole, it becomes clear that openings with a diameter less than $0.75 \mathrm{~mm}$ cause increased diffraction (3).

\section{METHODS}

Key in the therapeutic behavior of every patient with an irregular astigmatism is the preliminary analysis of the clinical case with a discussion of alternative methods for correction of visual acuity.

Furthermore, it has been shown that keratoconic eyes are more likely to develop cataracts and at a younger age than the general cataract population (4).

In 2014, a study was published to monitor the effectiveness of a new intraocular implant XtraFocus (Morcher GmbH, Germany) (5). It is an intraocular implant resembling an intraocular lens, with an occlusive area of $6.0 \mathrm{~mm}$, a central opening of $1.3 \mathrm{~mm}$ in diameter and no dioptric power. The innovative technology aims to correct irregular astigmatism of various etiologies. Thanks to its "2 Open-loop" technology of the haptics and the black hydrophobic material which XtraFocus is made of, the implant fits perfectly into the ciliary sulcus in pseudophakic eyes and makes it possible to perform the 'piggyback' configuration. The larger total diameter of the implant $(14 \mathrm{~mm})$, the 14-degree orientation of the haptics, as well as the rounded and polished edges, provide maximum prevention of a common complication of specifically positioned intraocular lenses (IOL) - Uveit-GlaucomaHyphema syndrome (6).

\section{CASE REPORT}

A 59-year-old man with severely reduced bestcorrected visual acuity of the right eye $\mathrm{VOD}_{5 \mathrm{~m}}$ $=0.1, \operatorname{VOD}_{33} \mathrm{~cm}=0.2$ (Snellen), due to observed keratoconus (refraction: -9. 25 D / $2.25 \mathrm{D} \mathrm{x} 71^{\circ}$ ) and mature cataract. The patient has a history of primary open-angle glaucoma.

A biomicroscopy of the right eye was performed, showing the presence of a transparent cornea and a mature cataract, which did not allow an examination of the fundus. (Figure 1)

From the performed B-scan there are no data for retinal detachment and the presence of other pathological changes in the fundus.

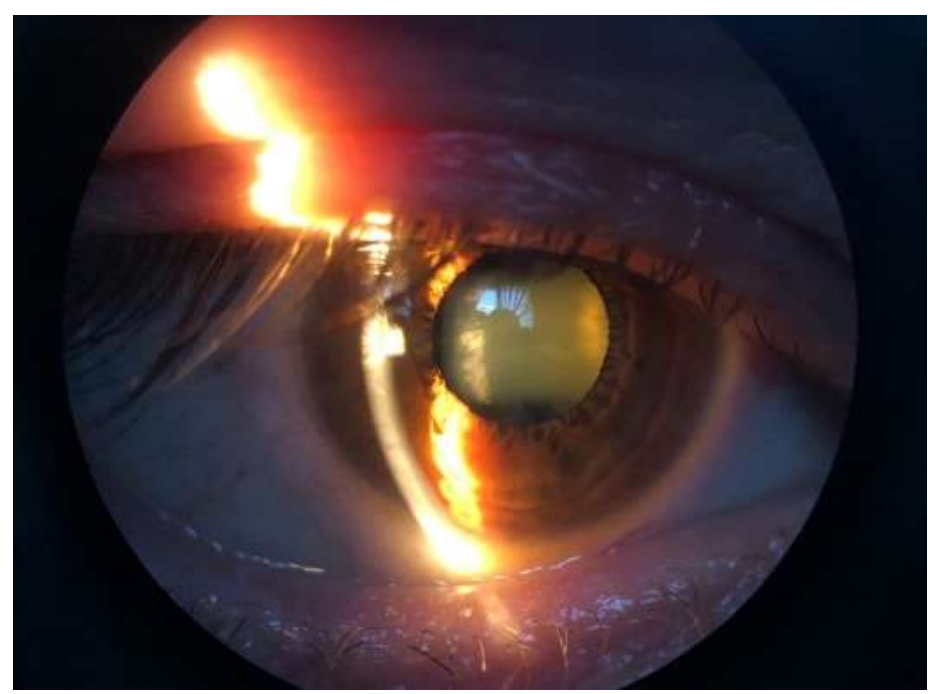

Figure 1. Biomicroscopy of the right eye with mature cataract;

\section{RESULTS}

Figure 1 clearly shows the astigmatism and the distinctive thinning of the cornea in the observed pathology. The shown optotype "E" serves to visualize the visual discomfort experienced by the patient in relation to the central 5-millimeter area of the cornea. (Figure 2)

The possibility of achieving optimal results in terms of contrast sensitivity and resolution after surgery was preliminarily analyzed and determined. The "Modulation Transfer Function" (MTF) indicator, determined in the central $1 \mathrm{~mm}$ area of the cornea in the pupil plane, was followed. It is the scientific tool for assessing the basic characteristics of the spatial resolution of an image system or its components. Two indicators are needed to determine the MTF: 1 . a measure of spatial detail called frequency, and 2 . a fundamental measure to determine conservation of this detail called modulation transmission shown in Figure 2 (7). (Figure 3) 
TSVETKOVA G., et al.

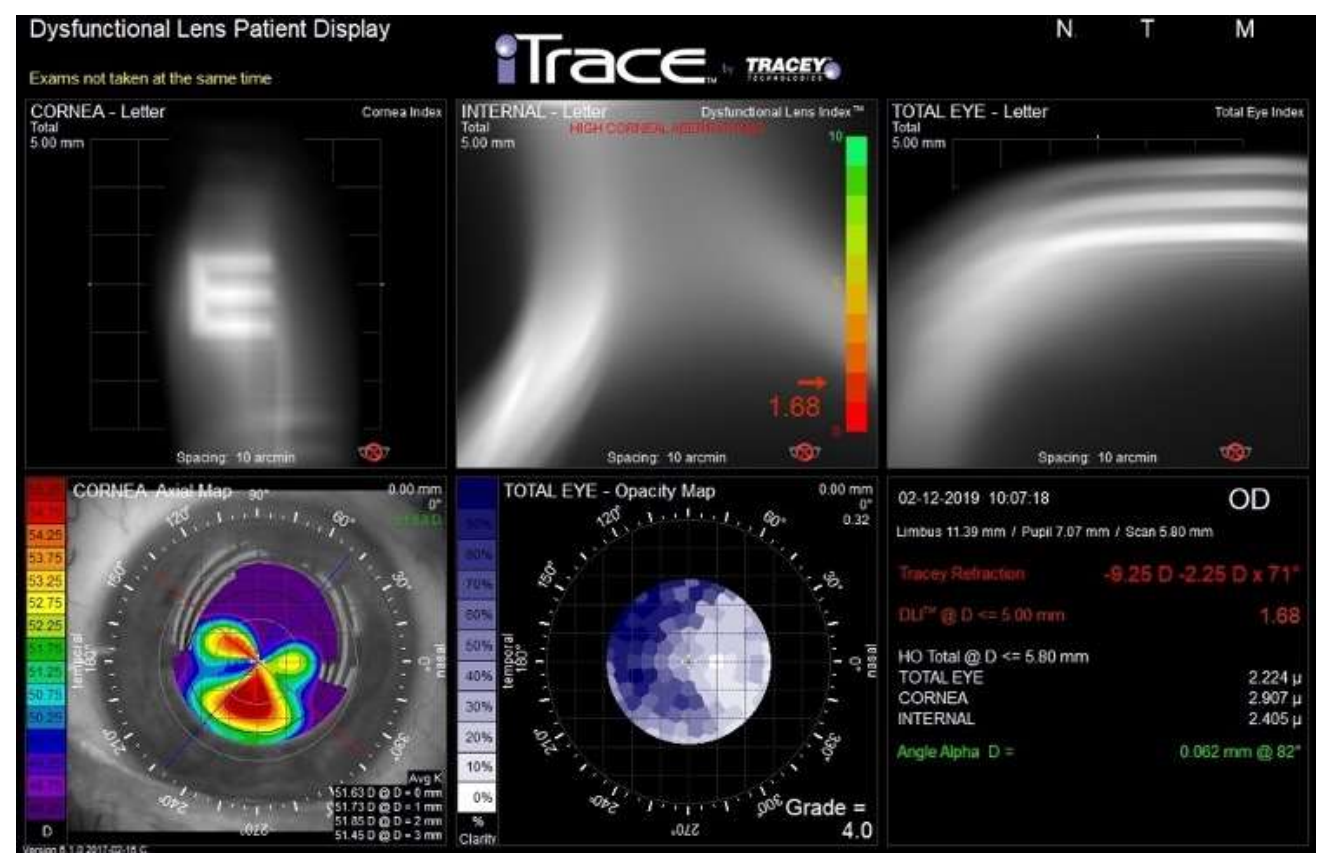

Figure 2. Determination of refraction and total visual acuity without correction for the central $5 \mathrm{~mm}$ area of the cornea by iTrace;

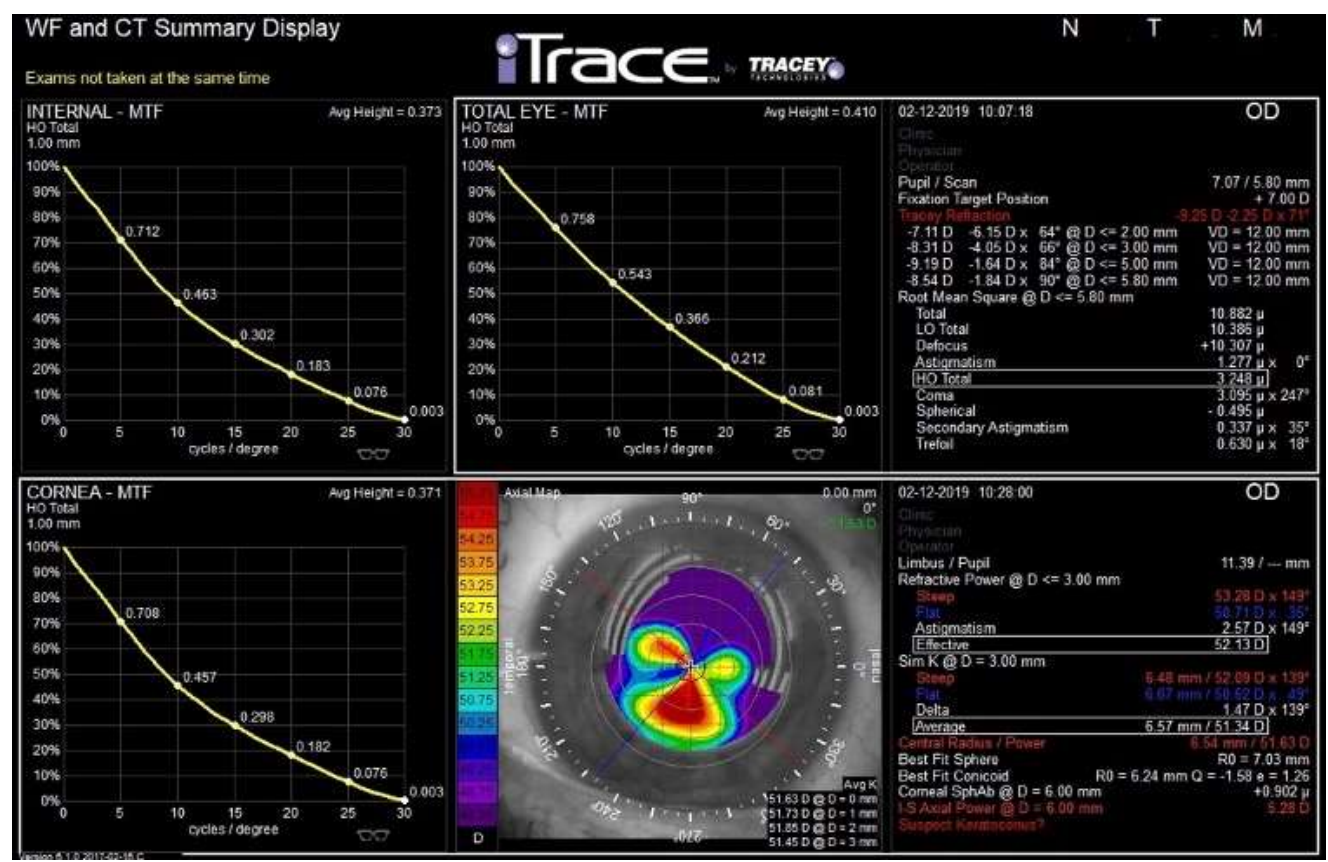

Figure 3. Determination of the MTF by iTrace;

The obtained measurements performed using an iTrace aberrometer and IOL Master 700 convincingly point to expected good results, committed to a satisfactory outcome for the patient and the surgeon.

Surgical treatment was performed to remove the cataract by standard phacoemulsification. Topical anesthesia (Proxymetacaine Hydrochloride $0.5 \%$ ) was used. Instead of implanting the IOL with optical power in the capsule bag (the use of such was not necessary in our case, due to the minimum refractive power, which was determined for it - +4.0 D), the pinhole implant XtraFocus was placed through a standard main incision of the cornea with dimensions $2.75 \mathrm{~mm}$ and a Medicel Viscojet injection system was used. Despite the small experience and the few cases of implantation of the pinhole in the sac, the early positive results after the procedure indicated the success of the risk that had been taken. (Figure 4) 
TSVETKOVA G., et al.

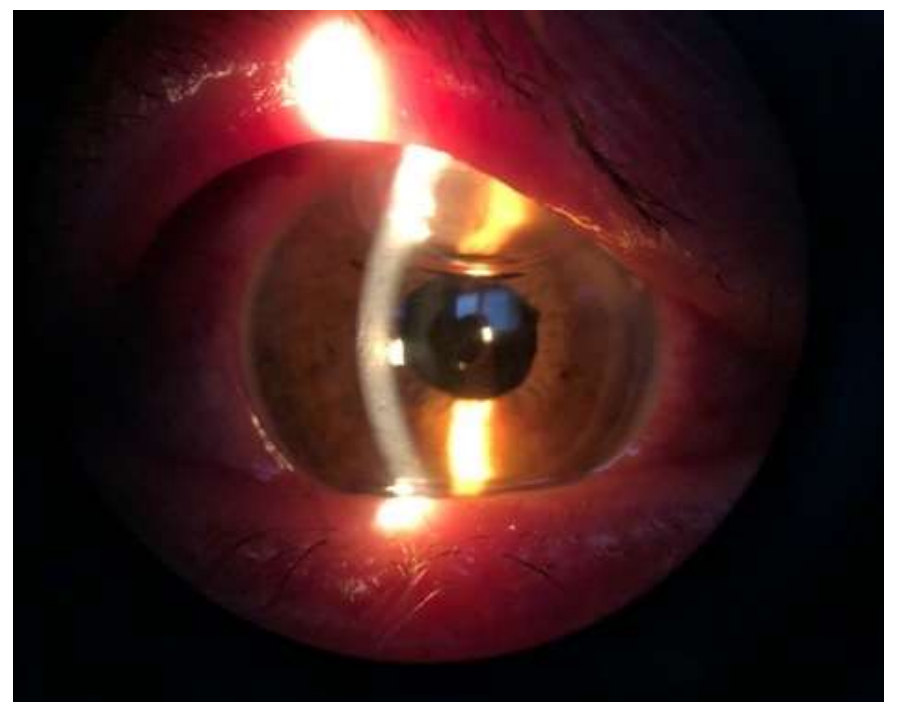

Figure 4. The right eye 24 hours after the surgery;

The patient's visual acuity, despite the presence of an air bubble in the upper $1 / 3$ of the anterior chamber, was determined as $\mathrm{VOD}_{5} \mathrm{~m}=0.5$ (Snellen). We prescribed topical conservative therapy for the postoperative period consisting of Ofloxacin $0.3 \%$ eye drops and Chloramphenicol $5 \mathrm{mg} / \mathrm{ml} /$ Dexamethasone 1 $\mathrm{mg} / \mathrm{ml}$ eye drops.

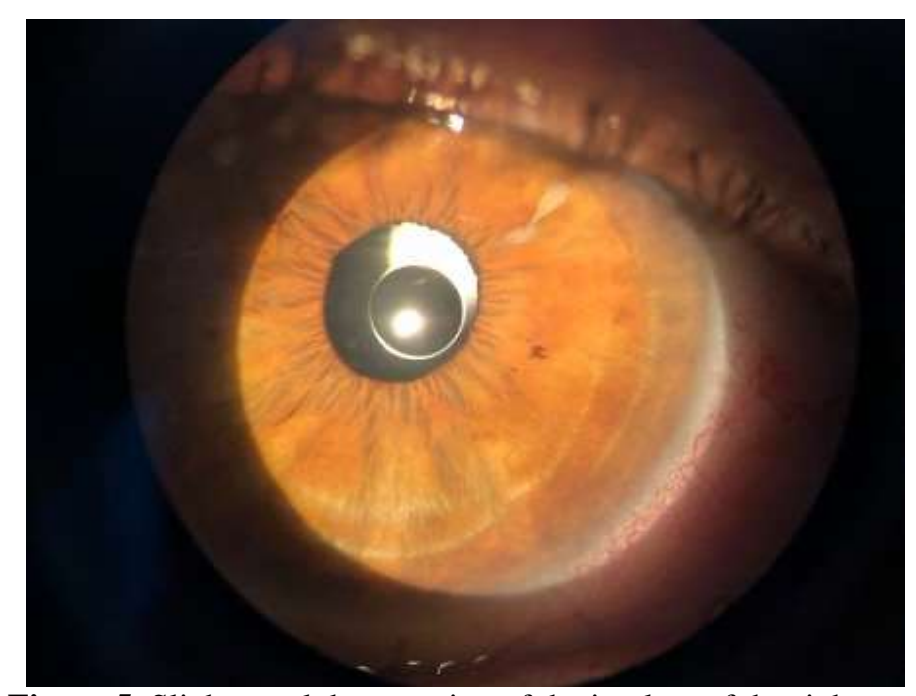

Figure 5. Slight nasal decentration of the implant of the right eye;

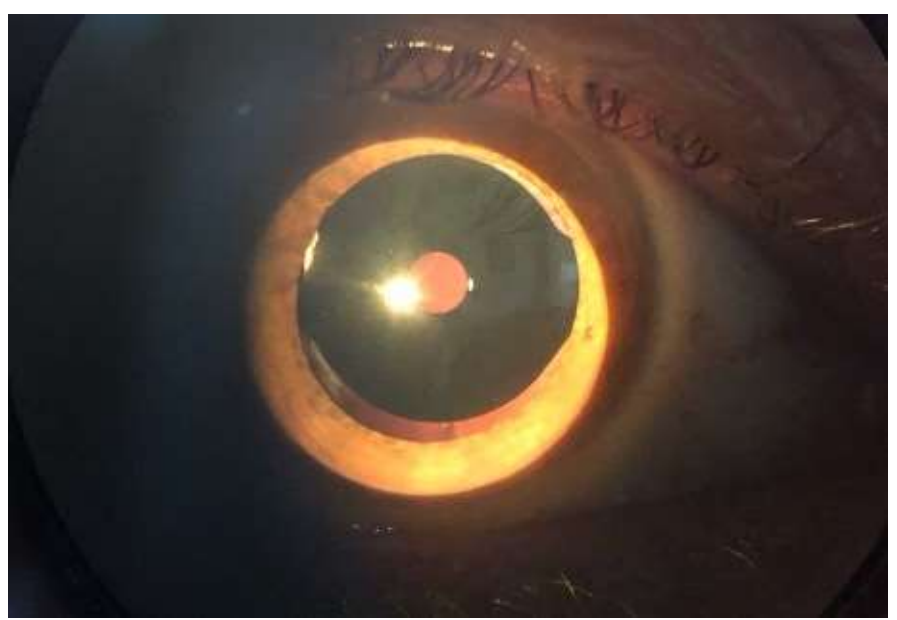

Figure 6. Mydriasis of the right eye; 
At a follow-up with the patient 6 months after surgery, visual acuity of the right eye was determined $\mathrm{VOD}_{5 \mathrm{~m}}$ and $33 \mathrm{~cm}=0.7$ (Snellen). During the biomicroscopy, the correct positioning of the implant with slight nasal decentration was established. (Figure 5 and Figure 6) According to the Stiles-Crawford effect (8), measurements showing the activity of the cones showed that those located noncentral to the pupillary plane, with a minimum nasal concentration of about $0.2-0.5 \mathrm{~mm}$, had a pronounced peak of photosensitivity (8).
TSVETKOVA G., et al.

Therefore, the nasal decentration of the implant is recommended and it is not a mistake in the performance of the surgical technique.

A standard automated perimetry (Humphrey 30-2) was also performed. (Figure 7)

There was a characteristic reduction in retinal photosensitivity, as well as an arcuate scotoma, most likely due to the accompanying eye disease, glaucoma.

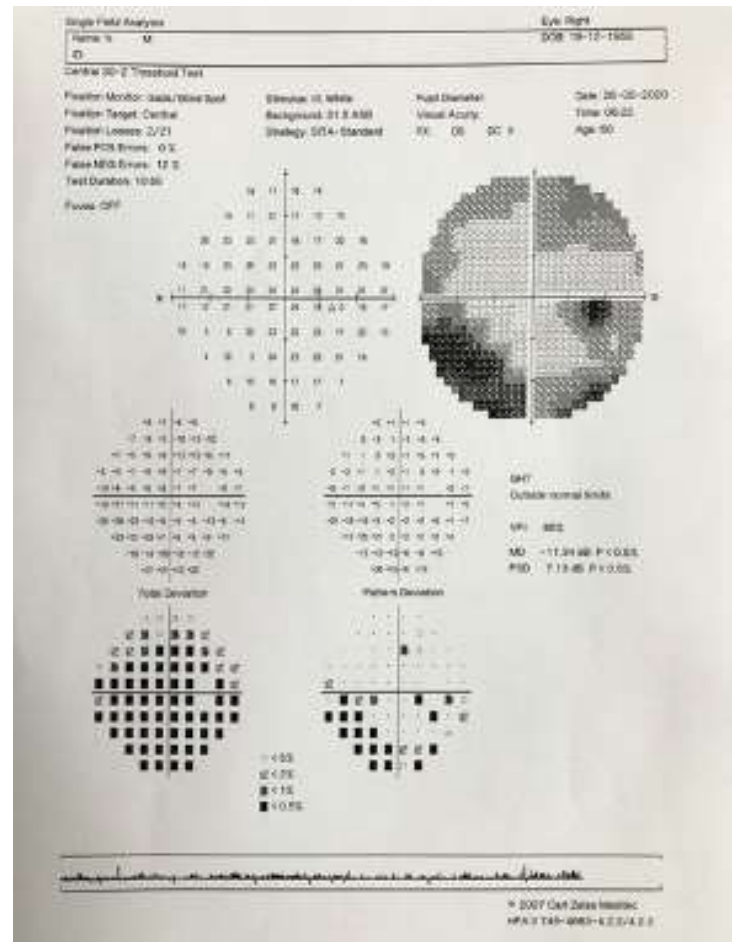

Figure 7. Standard Automated Perimetry (Humphrey 30-2), six months after the surgical treatment;

\section{DISCUSSION}

In 2017, the effect of XtraFocus implantation was evaluated in 21 patients aged between 35 and 85 years. Statistically, there was an improvement in the best corrected distance visual acuity. Good tolerance and a very satisfactory result in improving vision in patients with irregular astigmatism in keratoconus, radial keratotomy, penetrating keratoplasty and traumatic corneal laceration have been demonstrated (9).

Surgeons from the Trindade family, who invented the implant, published two cases of patients with irregular astigmatism and cataracts in 2019. Both were implanted with XtraFocus and had a capsulorhexis of the posterior lens capsule, with no decentration of the stenopic hole as a result. All results have proven extremely good with regards to visual acuity correction (10).

It is very important to mention that there are some disadvantages of the XtraFocus Implant like the limited ability to perform binocular indirect ophthalmoscopy and some treatments (e.g. retinal photocoagulation, pars plana vitrectomy) after implantation (6). Examination of the posterior segment is possible only with infrared-based imaging equipment because of the unique feature of the implant of being transparent to infrared light (6). Because of the restriction of the light entrance imposed by the pinhole aperture, a sensation of darkened vision may be reported after implantation but as long as the implant is well-centered it does not cause constriction of the visual field (6). 


\section{CONCLUSION}

XtraFocus is an intraocular implant that can be safely placed in the eye to correct high-grade irregular corneal astigmatism. The effectiveness of this new technology, using a principle from antiquity - pinhole, allows us to achieve results that fully meet the expectations of the patient and the surgeon, reaching visual acuity impossible in other circumstances. As pioneers for Bulgaria, we believe that our experience could stimulate surgeons to perform this type of surgery and take bolder decisions in order to improve the quality of life of the patient.

\section{REFERENCES}

1. Wittenberg S. Pinhole eyewear systems: a special report. J Am Optom Assoc. 64: 112-116, 1993.

2. Berman S. The coming revolution in lighting practice. Energy Users News. 25: 24-26, 2000.

3. Rubin ML. Quantification on the pinhole effect. Surv Ophthalmol. 21: 347-350, 1977.

4. Thebpatiphat N, Hammersmith KM, Rapuano CJ, Ayres BD, Cohen EJ. Cataract surgery in keratoconus. Eye Contact Lens: Sci Clin Pract. 33(5):244-246, 2007.
5. Trindade CLC, Trindade BLC. Novel pinhole intraocular implant for the treatment of irregular corneal astigmatism and severe light sensitivity after penetrating keratoplasty. J Cataract Refract Surg Online Case Rep. 3(1):4-7, 2015.

6. https://toomac.co.nz/uploads/8c696e27a8ce 032c72dd7b26d83bf6fa.pdf

7. "What is an MTF...and Why Should You Care" by Don Williams, Eastman Kodak, in RLG DigiNews, Vol. 2, No. 1, Feb. 15, 1998.

8. Stiles, W. S; Crawford, B. H. "The Luminous Efficiency of Rays Entering the Eye Pupil at Different Points". Proceedings of the Royal Society of London. Series B, Containing Papers of a Biological Character. 112 (778): 428-450, 1933.

9. Trindade CC, Trindade BC, Trindade FC, Werner L, Osher R, Santhiago MR. New pinhole sulcus implant for the correction of irregular corneal astigmatism. $J$ Cataract Refract Surg. 43(10):1297-1306, 2017.

10.Trindade BLC, Trindade FC, Trindade CLC. Intraocular pinhole implantation for irregular astigmatism after planned and unplanned posterior capsule opening during cataract surgery. $J$ Cataract Refract Surg. 45(3):372-377, 2019. 